\title{
A Higher Order Uniform Convergence Result for a Tuming Point Problem
}

\author{
H.-G. ROOS and R. VULANOVIĆ
}

We describe a new fitted scheme, of $k$-th order uniform accuracy with arbitrary $k$, for a turning point problem of cusp type. The scheme is constructed by applying an iterative technique to an auxiliary problem obtained after replacing coefficient functions in the original problem by piecewise polynomials. The analysis of the scheme is based on an improved stability result.

Key words: Singular perturbations, boundary value problems, turning points, higher order schemes AMS Subject Classifications: 34B15, 65L10

\section{Introduction}

Let us consider a turning point problem of the form

$$
\begin{aligned}
L u:=-\epsilon u^{\prime \prime}-x a(x) u^{\prime}+b(x) u & =f(x), \quad x \in(-1,1), \\
u(-1)=u(1) & =0,
\end{aligned}
$$

where $\epsilon$ is a small positive parameter and the functions $a, b$ and $f$ are sufficiently smooth. We assume that the coefficient of $u^{\prime}$ has a single simple zero and exclude any resonance phenomena. Thus, our additional assumptions are:

$$
\text { (i) } a(x) \geq \alpha>0 \quad \text { (ii) } \quad b(x) \geq 0, \quad b(0)>0 .
$$

Under these conditions, problem (1) admits a unique solution which satisfies the maximum principle and has a single, isolated turning point of cusp type at $x=0$.

Abrahamsson [1] has derived the asymptotic behaviour of the solution as $\epsilon \rightarrow 0$. The unique solution of (1) converges to the solution of the reduced equation which satisfies both boundary conditions. The general solution of the reduced equation admits the following representation:

$$
v(x)=w(x)+ \begin{cases}c_{1}|x|^{\lambda} \exp \left(\int_{0}^{x} \psi(t) d t\right) & \text { if } x<0 \\ c_{2} x^{\lambda} \exp \left(\int_{0}^{x} \psi(t) d t\right) & \text { if } x>0\end{cases}
$$

where $\lambda=b(0) / a(0)$, and $w$ and $\psi$ are some smooth functions (see Lemma 3.1 in [1]). The constants $c_{1}$ and $c_{2}$ are determined by the boundary conditions in the limit of $u$ as $\epsilon \rightarrow 0$. A priori estimates of the derivatives of the exact solution have been obtained in [2]:

$$
\left|u^{(l)}(x)\right| \leq C\left(x^{2}+\epsilon\right)^{\frac{\lambda-t}{2}}, \quad l=0,1, \ldots
$$

H.-G. Roos: Techn. Universität, Inst. für Numer. Math., Mommsonstr. 13, D - 01069 Dresden R. Vulanovic: University of Novi Sad, Inst. Math., Trg D. Obradovica 4, YU - 21000 Novi Sad 
The representation (3) and the sharp estimates (4) are the main reason that the standard discretization methods fail at least in the most complicated situation $0<\lambda<1$. Let us generally assume that

$$
0<\lambda<1 \text {, }
$$

even though our method works for every $\lambda>0$. Under the assumption (5), the well known El-Mistikawy-Werle scheme has been shown to be of order $O\left(h^{\lambda}\right)$, uniformly with respect to the parameter $\epsilon$ (see section 3 in [2]). Farrell [4] has given sufficient conditions for a class of finite-difference schemes (including the standard upwinding) to have the same uniform rate of convergence in the maximum norm.

The accuracy $O\left(h^{\lambda}\right)$ is somewhat unsatisfactory, but Farrell and Gartland [5] were able to construct a uniform $O(h)$-scheme. In this paper we shall show that their approach can be regarded as the first step of an iterative process which yields $O\left(h^{k}\right)$-accuracy in the maximum norm for arbitrary $k$. This result is based on a new stability estimate. The iterative process is used for handling an auxiliary problem with piecewise polynomial coefficients. This is similar to the technique from [3], where singular perturbation problems without turning points have been treated. Another approach for numerical treatment of singular perturbation problems is to use the classical finite-difference schemes on special discretization meshes which are dense in the layers. The first result by this approach for the problem (1) has been given by Liseikin [6]. It was later improved in [10], [7] and finally in [11]. The papers [6], [7] and [10] give uniform methods of first order, while a uniform second order result has been proved in [11]. Thus, our higher order uniform convergence result for turning point problems is new.

Since the main purpose of this paper is to introduce a technique by which the method from [5] can be improved, we decided to present no numerical results.

\section{The Farrell-Gartland Approach}

Let some grid $-1=x_{0}<x_{1}<\cdots<x_{N}=1$ be given with $h_{i}=x_{i+1}-x_{i}$ and the mesh width $h=\max h_{i}$. We define $\bar{a}$ as a piecewise constant approximation to $a$ :

$$
\bar{a}(x)=\bar{a}_{i} \text { on }\left(x_{i}, x_{i+1}\right) \text { with }\|a-\bar{a}\|_{\infty} \leq C h .
$$

Here and throughout the paper $C$ denotes a generic constant independent of $\epsilon$ and $h$. The functions $b$ and $f$ are approximated in the same way. Then, Farrell and Gartland's basic idea is to define the approximate solution $u_{h}$ as the solution to the boundary value problem

$$
\begin{aligned}
\bar{L} u_{h}:=-\epsilon u_{h}^{\prime \prime}-x \bar{a}(x) u_{h}^{\prime}+\bar{b}(x) u_{h} & =\bar{f}(x), \quad x \in(-1,1), \\
u_{h}(-1)=u_{h}(1) & =0 .
\end{aligned}
$$

Farrell and Gartland use the stability result for $\vec{L}$

$$
\|v\|_{\infty} \leq C\|\bar{L} v\|_{\infty}
$$

to estimate the error $u-u_{h}$. Since

$$
\bar{L}\left(u-u_{h}\right)=f-\bar{f}+(\bar{b}-b) u-(\bar{a}-a) x u^{\prime},
$$

the stability estimate (7) yields

$$
\left\|u-u_{h}\right\|_{\infty} \leq C\left(\|f-\bar{f}\|_{\infty}+\|b-\bar{b}\|_{\infty}+\|\bar{a}-a\|_{\infty}\left\|x u^{\prime}\right\|_{\infty}\right) .
$$

Then (4) implies that

$$
\left\|x u^{\prime}\right\|_{\infty} \leq C \max _{x \in[-1,1]}\left\{|x|\left(x^{2}+\epsilon\right)^{\frac{\lambda-1}{2}}\right\} \leq C,
$$


and the desired first order uniform convergence result follows from (8):

$$
\left\|u-u_{h}\right\|_{\infty} \leq C h .
$$

For our approach we need some improvement of the stability result (7) as well as the error estimate (9). First, we announce the improved stability result.

Lemma 1. Under the conditions (2), the stability estimate $\|v\|_{\infty}+\left\|x v^{\prime}\right\|_{\infty} \leq C\|\bar{L} v\|_{\infty}$ holds for $h \leq h_{0}$, where $h_{0}$ is sufficiently small but independent of $\epsilon$.

Further on, we shall always assume that $h \leq h_{0}$. We will prove Lemma 1 in the appendix. Applying Lemma 1 we get a sharpened version of the corresponding theorem in [5]:

Theorem 1. Let the approximate problem (6) be constructed by replacing the functions $a, b, f$ from (1) by functions $\bar{a}, \bar{b}, \bar{f}$ satisfying $\|\bar{a}-a\|_{\infty}+\|b-\bar{b}\|_{\infty}+\|f-\bar{f}\|_{\infty} \leq C h$. Then the error between the solutions to the original problem (1) and to the approximate problem (6) satisfies

$$
\left\|u-u_{h}\right\|_{\infty}+\left\|x\left(u-u_{h}\right)^{\prime}\right\|_{\infty} \leq C h .
$$

\section{The New Higher Order Scheme}

On the given grid we approximate $a$ by $\bar{a}$, a piecewise polynomial of degree $k$, such that

$$
\begin{gathered}
\bar{a}=\sum_{\mu=0}^{k} a_{\mu}, \quad\left\|a-\sum_{\mu=0}^{l} a_{\mu}\right\|_{\infty} \leq C h^{l+1} \text { for } l=0(1) k, \\
\left\|a_{\mu}\right\|_{\infty} \leq C h^{\mu} \text { for } \mu=0(1) k .
\end{gathered}
$$

We shall use analogous approximations to $b$ and $f$ as well. Further, we introduce the abbreviations

$$
\bar{L}_{0} v:=-\epsilon v^{\prime \prime}-x a_{0} v^{\prime}+b_{0} v \text { and } \bar{L}_{l} v:=-x a_{l} v^{\prime}+b_{l} v \text {. }
$$

Theoretically, we could introduce an approximation $u_{h}^{*}$ which solves the problem

$$
\begin{aligned}
-\epsilon w^{\prime \prime}-x \bar{a}(x) w^{\prime}+\bar{b}(x) w & =\bar{f}(x), \quad x \in(-1,1), \\
w(-1)=w(1) & =0 .
\end{aligned}
$$

In the same way as in Section 2, we are able to prove $\left\|u-u_{h}^{*}\right\|_{\infty} \leq C h^{k}$, but this result is practically worthless because it is impossible to handle equations with piecewise polynomial coefficients of higher degree.

Therefore we introduce the following iterative process:

$$
\begin{aligned}
& \bar{L}_{0} u_{h}^{0}=f_{0}, \quad u_{h}^{0}(-1)=u_{h}^{0}(1)=0, \\
& \bar{L}_{0} u_{h}^{i+1}=\sum_{l=0}^{i+1} f_{l}-\sum_{l=1}^{i+1} \bar{L}_{l} u_{h}^{i}, \quad u_{h}^{i+1}(-1)=u_{h}^{i+1}(1)=0, \quad i=0(1) k-1 .
\end{aligned}
$$

Thus $u_{h}^{0}, u_{h}^{1}, \ldots, u_{h}^{k}$ solve differential equations with the same left hand side as in the FarrellGartland approach.

We analyse the method by mathematical induction starting with $u_{h}^{0}$ which is the same as $u_{h}$ from Theorem 1, and thus satisfies

$$
\left\|u-u_{h}^{0}\right\|_{\infty}+\left\|x\left(u-u_{h}^{0}\right)^{\prime}\right\|_{\infty} \leq C h .
$$


Then for $i \geq 0$, we have

$$
\begin{aligned}
\bar{L}_{0}\left(u_{h}^{i+1}-u\right) & =\sum_{l=0}^{i+1} f_{l}-\sum_{l=1}^{i+1} \bar{L}_{l} u_{h}^{i}-L u+\left(L-L_{0}\right) u \\
& =\left(\sum_{l=0}^{i+1} f_{l}-f\right)-\sum_{l=1}^{i+1} \bar{L}_{l} u_{h}^{i}+(b-\bar{b}) u-(a-\bar{a}) x u^{\prime}+\sum_{l=1}^{k} \bar{L}_{l} u
\end{aligned}
$$

thus

$$
\vec{L}_{0}\left(u_{h}^{i+1}-u\right)=\left(\sum_{l=0}^{i+1} f_{l}-f\right)+\sum_{l=1}^{i+1} \bar{L}_{l}\left(u-u_{h}^{i}\right)+(b-\bar{b}) u-(a-\bar{a}) x u^{\prime}+\sum_{l=i+2}^{k} \bar{L}_{l} u .
$$

By applying our stability result from Lemma 1 and the uniform boundedness of $\|u\|_{\infty}+\left\|x u^{\prime}\right\|_{\infty}$, we obtain

$$
\left\|u-u_{h}^{i+1}\right\|_{\infty}+\left\|x\left(u-u_{h}^{i+1}\right)^{\prime}\right\|_{\infty} \leq C\left\{h^{i+2}+h\left(\left\|u-u_{h}^{i}\right\|_{\infty}+\left\|x\left(u-u_{h}^{i}\right)^{\prime}\right\|_{\infty}\right)+h^{k+1}\right\} .
$$

So, we have proved the following theorem by mathematical induction.

Theorem 2. The error between the solutions to the original problem (1) and to the $i$-th iteration of the process (10) satisfies

$$
\left\|u-u_{h}^{i}\right\|_{\infty}+\left\|x\left(u-u_{h}^{i}\right)^{\prime}\right\|_{\infty} \leq C h^{i+1}, \quad i=0(1) k,
$$

thus $u_{h}^{i}$ is an $O\left(h^{i+1}\right)$ uniform approximation to $u$.

Finally, let us remark that there exist well known procedures to handle the problem

$$
\bar{L}_{0} u_{h}=g, \quad u_{h}(-1)=u_{h}(1)=0,
$$

in an effective way (using patched spline functions [8], local Green's functions, or Marchuk type integral relations [3], [5]). They all lead to tridiagonal linear systems which have nice properties (their matrices are $\mathrm{M}$-matrices).

\section{Appendix: Proof of the Stability Estimate}

We consider the boundary value problem (using the notation of Section 2 again)

$$
\begin{aligned}
\bar{L} v=-\epsilon v^{\prime \prime}-x \bar{a}(x) v^{\prime}+\bar{b}(x) v & =f(x), \quad x \in(-1,1), \\
v(-1)=v(1) & =0,
\end{aligned}
$$

and try to estimate $x v^{\prime}$ in terms of $F$, where $\|v\|_{\infty} \leq C F, \quad F=\|f\|_{\infty}=\|\bar{L} v\|_{\infty}$. Introducing the integrating factor $e^{\phi(x)}$ with $\epsilon \phi(x)=\int_{0}^{x} \bar{a}(t) t d t$, we obtain

$$
\epsilon\left(e^{\phi} v^{\prime}\right)^{\prime}=(\bar{b} v-f) e^{\phi}, \quad \text { or } \quad x v^{\prime}(x)=S_{1}+S_{2},
$$

where

$$
S_{1}=x v^{\prime}(0) e^{-\phi(x)} \quad \text { and } \quad S_{2}=\frac{x}{\epsilon} \int_{0}^{x}(\bar{b}(t) v(t)-f(t)) e^{\phi(t)-\phi(x)} d t .
$$

Let us start estimating $S_{2}$. We get

$$
\left|S_{2}\right| \leq \frac{C F}{\epsilon}\left|x \int_{0}^{x} e^{\phi(t)-\phi(x)} d t\right| .
$$


Let us assume $x>0$ (the case $x<0$ can be handled analogously). By taking into account that $\bar{a}(x) \geq \alpha>0$ for $h \leq h_{0}$, we get, for $0 \leq t \leq x$,

$$
\phi(t)-\phi(x)=\frac{1}{\epsilon} \int_{x}^{t} \bar{a}(s) s d s \leq \frac{\alpha}{2 \epsilon}\left(t^{2}-x^{2}\right)=\frac{\alpha}{2 \epsilon}(t-x)(t+x) \leq \frac{\alpha}{2 \epsilon} x(t-x)
$$

and obtain

$$
\left|S_{2}\right| \leq C F \frac{x}{\epsilon} \int_{0}^{x} e^{\frac{a x}{2 t}(t-x)} d t \leq C F .
$$

Let us now estimate $S_{1}$. We only have to prove

$$
\left|v^{\prime}(0)\right| \leq C F / \sqrt{\epsilon},
$$

since this inequality and $e^{-\phi(x)} \leq e^{-\frac{\alpha x^{2}}{2 e}}$ imply

$$
\left|S_{1}\right| \leq C F \frac{1}{\sqrt{\epsilon}}\left|x e^{-\frac{a x^{2}}{2 \epsilon}}\right| \leq C F \text {. }
$$

Let us prove (11). We choose $x^{*} \in(0, \sqrt{\epsilon})$ such that $v^{\prime}\left(x^{*}\right)=(v(\sqrt{\epsilon})-v(0)) / \sqrt{\epsilon}$. Then from $(i)$ we conclude

$$
\left|v^{\prime}\left(x^{\bullet}\right)\right| \leq C F / \sqrt{\epsilon}
$$

Integrating the differential equation from 0 to $x^{*}$ we get

$$
-\epsilon v^{\prime}\left(x^{*}\right)+\epsilon v^{\prime}(0)-\int_{0}^{x^{*}} \bar{a}(t) t v^{\prime}(t) d t=\int_{0}^{x^{*}}(f(t)-\bar{b}(t) v(t)) d t
$$

wherefrom we obtain the inequality

$$
\left|v^{\prime}(0)\right| \leq\left|v^{\prime}\left(x^{*}\right)\right|+\frac{1}{\epsilon}\left|\int_{0}^{x^{*}} \bar{a}(t) t v^{\prime}(t) d t\right|+\frac{1}{\epsilon}\left|\int_{0}^{x^{*}}(f(t)-\bar{b}(t) v(t)) d t\right| .
$$

Due to (12) and $x^{*} \in(0, \sqrt{\epsilon})$ we get

$$
\left|v^{\prime}(0)\right| \leq \frac{C F}{\sqrt{\epsilon}}+\frac{S_{3}}{\epsilon}, \text { where } S_{3}=\left|\int_{0}^{x^{\cdot}} \bar{a}(t) t v^{\prime}(t) d t\right| .
$$

Let us assume $x^{*} \in\left(x_{k}, x_{k+1}\right]$. Then we have

$$
S_{3}=\left|\sum_{i=0}^{k} \bar{a}_{i} \int_{x_{i}}^{\dot{x}_{1+1}}\left[(x v)^{\prime}-v\right] d x\right| \quad \text { with } \quad \hat{x}_{l}=\left\{\begin{array}{lll}
x_{l} & \text { if } l \leq k, \\
x^{*} & \text { if } \quad l=k+1 .
\end{array}\right.
$$

This representation leads to

$$
S_{3} \leq \sum_{i=0}^{k} \bar{a}_{i}\left|\int_{x_{i}}^{\dot{x}_{i+1}} v d x\right|+\left|\sum_{i=0}^{k} \bar{a}_{i}\left[\dot{x}_{i+1} v\left(\dot{x}_{i+1}\right)-x_{i} v\left(x_{i}\right)\right]\right| .
$$

The first term is immediately bounded by $C F \sqrt{\epsilon}$. The second term can be written in the form

$$
\left|\sum_{i=1}^{k}\left(\bar{a}_{i+1}-\bar{a}_{i}\right) x_{i} v\left(x_{i}\right)+\bar{a}_{k} \hat{x}_{k+1} v\left(\dot{x}_{k+1}\right)\right| \text {, }
$$

thus, $C F \sqrt{\epsilon}$ is an upper bound again. Therefore we obtain $S_{3} \leq C F \sqrt{\epsilon}$, and finally from the inequality (13) $\left|v^{\prime}(0)\right| \leq C F / \sqrt{\epsilon}$. Thus, Lemma 1 is proved.

The technique used here is essentially the one introduced in [6]. We made some necessary modifications to handle the piecewise constant coefficient $\mathbf{a}$.

Acknowledgements: A part of the first author's work and the second author's entire work on this paper were done during their visit to the Department of Mathematice and Computer Science at Kent State University, Kent, Obio, U.S.A. The authors would like to use this opportunity to thank Professot O. P. Stackelberg, Chairman, and Professors P. A. Farrell and E. C. Gartland for their hospitality. 


\section{References}

(1] ABRAHAMSSON, L.R.: A priori estimates for the solutions of singular perturbations with a turning point. Stud. Appl. Math. 56 (1977),51-69.

[2] BERGER, A.E., HAN, H. and R.B. KELLOGG: $A$ priori estimates and analysis of a numerical method for a turning point problem. Math. Comput. 42 (1984),465-492.

[3] BOGLAEV, I.P. and V.V. SIROTKIN: $A$ uniform iterative method for singular perturbation problems. In: Num. Methods in Singularly Perturbed Problems (eds.: H.-G. Roos et al.). Techn. Univ. Dresden 1991, pp. 13-26.

[4] FARRELL, P.A.: Sufficient conditions for the uniform convergence of difference schemes for a singularly perturbed turning point problem. SIAM J: Numer. Anal. 25 (1988), 618-643.

[5] FARRELL, P.A. and E.C. GARTLAND: $A$ uniform convergence result for a turning point problem. In: Proc. BAIL 5 Conference (eds.: Guo Ben-yu et al.). Dublin: Boole Press 1988, pp. 127-132.

[6] LISEIKIN, V.D.: Numerical solution of a second-order ordinary differential equation with a small parameter multiplying the highest derivative (in Russian). Chisl. Metody Mekh. Sploshn. Sredy 13 (1982), 71-80.

[7] LISEIKIN, V.D.: Numerical solution of a singularly perturbed equation with a turning point (in Russian). Zh. Vychisl. Mat. i Mat. Fiz. 24 (1984), 1812-1818.

[8] ROOS, H.-G.: Global uniformly convergent schemes for a singularly perturbed boundary value problem using patched base spline functions. J. Comp. Appl. Math. 29 (1990), 69-77.

[9] ROOS, B.-G. and M. STYNES: $A$ uniformly convergent discretization method for a fourth order singular perturbation problem. Bonner Math. Schriften 228 (1991), 30-40.

[10] VULANOVIĆ, R.: Mesh construction for numerical solution of a type of singular perturbation problems. In: Numerical Methods and Approximation Theory (ed.: G.V. Milovanović). Niš: University of Nis̃ 1984, pp. 137-142.

[11] VULANOVIĆ, R.: A second order uniform numerical method for a turning point problem. Univ. u Novom Sadu, Zb. Rad. Prirod.-Mat. Fak. Ser. Mat. 18 (1988), 17-30. 